\title{
Cyclic Wiener Filtering Algorithm in Discrete Cosine Transform Domain for Vibration Signal
}

\author{
Zhang Feng ${ }^{1}$, Yang Shangjun ${ }^{2}$, Hu Yantao $^{3}$ and Shi Xianfeng ${ }^{4}$ \\ ${ }^{1}$ Associate Professor, Xi'an Technological University, China \\ ${ }^{2}$ Master Graduate student, Xi'an Technological University, China \\ ${ }^{3}$ Master Graduate student, Xi'an Technological University, China \\ ${ }^{4}$ Lecturer, Xi'an Technological University, China
}

\begin{abstract}
In order to solve the problem that the effect of using cyclic Wiener filter directly to remove the noise on the non-stationary vibration signal is poor, the paper applies discrete cosine transform to the cyclic Wiener filter, proposing the cyclic Wiener filtering algorithm in discrete cosine transform domain for the vibration signal. Using the energy concentration characteristic of discrete cosine transform and the linear phase characteristic of cyclic Wiener filtering, the paper adopts the method of combining both of them with segmented processing to give full play to the performance of the Wiener filter and achieves a better filtering effect with a lower filter order. Combining with industrial field turbine vibration signal, paper makes a simulation analysis for this algorithm. The result of simulation shows that the algorithm has a good noise filtering effect, and the filtered signal has no obvious phase distortion. So, the algorithm is suitable for removing noise on vibration signal and the filtering effect is better than just using cyclic Wiener filter or DCT filter only.
\end{abstract}

\section{Introduction}

It has the magnificent meaning to insure the safety and stable operation for mechanical equipment, which is based on the analysis of vibration signal with the advantage of online, true time, non-ablative, convenient and accurate diagnose for mechanical fault diagnosis ${ }^{[1]}$. On the process of acquirement and transmit for the vibration signal, it is unavoidable to be disturb by the noise of environment and equipment itself. So that the operation status of rotating machinery cannot be truly reflected, which influence the analysis and dispose in the later stage, even maybe cause the erroneous judgment ${ }^{[2]}$. So to require accurate character of vibration signal and using it to detect and diagnose the fault for the turbine running state, the priority is to denoise the vibration signal.

For the common filter technique, it is difficult to balance the quality between filtering effect and linear phase. Whereas, the linear phase of vibration signal filtering is strict, this is not allowed to produce the phase distortion clearly. Document[3] uses cyclic Wiener filtering to filter the self-adaption noise for the vibration signal, and it achieves the better effect, but it has a bad effect by using the cyclic Wiener filtering directly to filter non-stable random signal, meantime, the self-adaption filtering algorithm is complicated and the computation is heavy; The method of non-phase deviation filtering in DCT domain for vibration signal, presented by document[4], not only can guarantee the signal's phase character, but also achieves the reduction of data size, however, the filtering in DCT domain has a question that the filtering effect is bad while processing the long data. Soft threshold wavelet filtering for vibration signal, which is presented in document [5], achieves a good effect in filtering, but this algorithm needs to estimate the noise's square error, also it has difficulty in choosing the threshold. The paper on the basis of analyzing the vibration signal character and filter requirement, puts forward to cyclic Wiener filtering algorithm in Discrete Cosine Transform Domain for vibration signal, to guarantee the better filtering effect on the phase character, meanwhile achieves the data compression.

\section{Vibration signal sampling and filtering requirement}

\subsection{Vibration signal sampling}

In order to make this research and analysis more targeted, we sample the turbine vibration signal in industrial scene. The parameter of data sampling is:32 frequency multiplication sampling, rotating speed of 32 times, with the cooperation for the back of the FFT calculation, sampling points are set to 2 of the whole power, $N=2^{M}$.Also for the facilitate processing, each channel samples 128 dots continuously. The sampling signal waveform is shown as Figure 1. In order to research the denoising performance, adding the random 
noise to the signal, vibration signal after adding noise is shown as figure $2^{[6]}$.

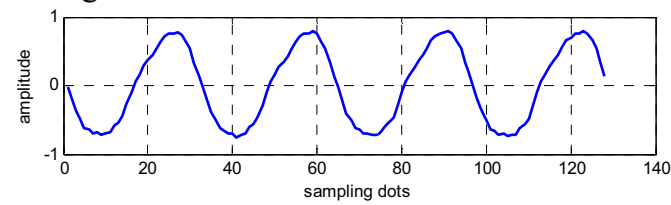

Figure1. Original sampling of vibration signal

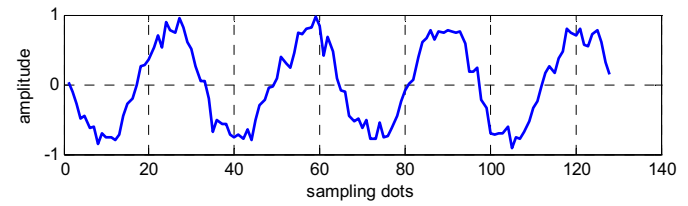

Figure2. Vibration signal after being added noise

\subsection{Vibration signal filtering requirement}

In order to acquire turbine's running status accurately, two basic requirements should be met for the turbine's vibration signal denoising as follow;

(1) The information of each harmonic phase for the vibration signal includes shaft vibration's position information. So after denoising, the signal should not produce phase distortion or less phase distortion. The nonlinear of phase frequency characteristic for filtering algorithm may cause the waveform distortion, and this kind of distortion is a manifestation of phase distortion. So this requires that filtering algorithm should have a good linear phase property.

(2) Under the premise of ensuring the phase characteristics, it is better to filter the noise to obtain the vibration signal that can truly reflect the turbine's operation. Especially in the following-up spectral estimation for vibration signal. The noise will have a great influence on variance performance for spectral estimation algorithm and the accuracy for the spectral estimation. So removing the noise is as much as possible.

\section{Signal's filtering processing in DCT Domain}

\subsection{Discrete Cosine Transform}

Discrete cosine transform is a kind of orthogonal transform in the real number field. For the sequence $x(n)$, the length of $N$.The discrete cosine transform is represented by $x_{c}(k)$, define:

$$
\begin{array}{r}
X_{c}(k)=\operatorname{DCT}\left[x(n)=\sqrt{\frac{2}{N}} c(k) \sum_{n=0}^{N-1} x(n) \cos \left[\frac{(2 n+1) k \pi}{2 N}\right]\right. \\
\text { Where: } \quad c(k)=\left\{\begin{array}{cc}
\frac{1}{\sqrt{2}}, & k=0 \\
1, & 1 \leq k \leq N-1
\end{array}\right.
\end{array}
$$

The inverse transform is:

$$
x(n)=I D C T\left[X_{c}(k)\right]=\sqrt{\frac{2}{N}} \sum_{n=0}^{N-1} c(k) X_{c}(k) \cos \left[\frac{(2 n+1) k \pi}{2 N}\right]
$$

DCT has the property of solution correlation in large degree, after the transforming, the correlation of the signal becomes reduced. Most of energy for signal only concentrates on a few of transformation coefficients also these transformation coefficients represent signal's lower frequency energy. So abandoning the following data can still keep the signal's characteristics. Also the noise of the mixed coefficient is removed; meanwhile, the data volume is reduced and compressed. The main process of denoising by using DCT is: input the data then transform DCT by Formula (1); According to the characteristic of energy concentration, retain the previous data, abandon the behind data, then to fill in the data of 0 to the original data length; By formula (3) DCT inverse transform for the data, then obtain the new data after filtering.

\subsection{DCT filtering effect analysis}

Signal denoising based on DCT is a filtering method of non-phase deviation. But the filtering effect depends on the number of reserved coefficient which is in DCT domain. Using this algorithm to filter the noisy vibration signal then compared to the original signal, the result shows in Figure 3.

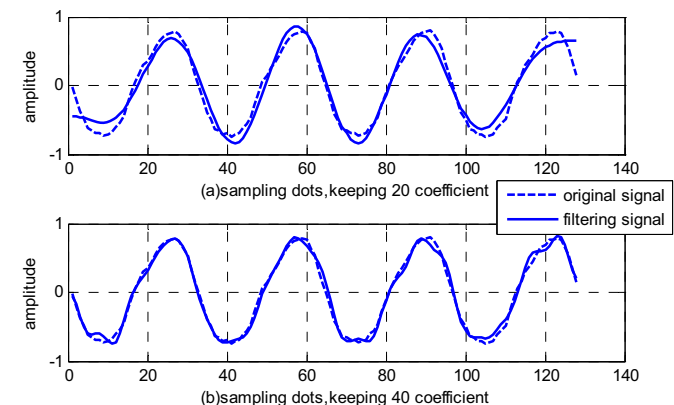

Figure3. Turbine Vibration signal after being filtered by DCT

The Figure3 (a) shows that when the coefficient of retention is low, the noise can be filtered in large degree, but after filtering the waveform is too smooth, which generates the obvious distortion. The reason is that while using DCT filtering to abandon the behind data, if the retained data is too less, then the lost energy is too much, after restoration, the original signal cannot be presented very well.

The Figure3 (b) shows that when the coefficient is more, the filtering effect becomes worse obviously. A lot of noise and disturbance can't be effectively removed. For DCT filtering, the latter coefficients which containing the adding noise is abandoned, whereas the former coefficients which the containing the noise is restrained completely.

Although applying the DCT to turbine vibration signal filtering, which can take effects in some degree, this algorithm is a kind of non-linear filtering, it needs to choose the proper length for keeping, if the abandoned data is too less, the noise cannot be removed effectively; if the abandoned data is too more, the signal's information for itself will loss too much; In addition, when the length of signal sampling is long, this choice of method will be much more difficult. So this algorithm directly using for vibration signal denoising still have some kind of problems, for the long data, it is better to deal with it by the method of periodic segment before dealing ${ }^{[7-8]}$.

\section{Cyclic Wiener Filtering Algorithm}

Wiener filter is the linear filter which is based on the minimum mean square error. So it can be designed as finite 
duration impulse response or infinite duration impulse. Taking the FIR Wiener filter as an example, basic principle is shown in Figure 4.

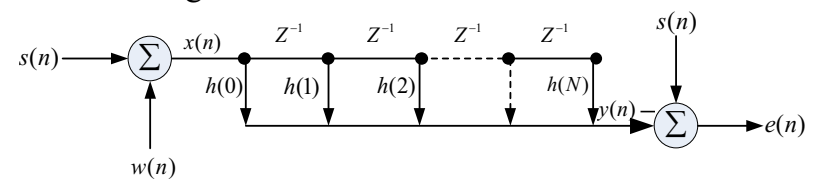

Figure4. Wiener filter schematic

In Figure 4, $s(n)$ is the real signal, $w(n)$ is the additive noise or measurement error, $x(n)$ is the observed signal, $h(n)$ is the Wiener filtering output signal, $e(n)$ is the processing error. By Figure 4, we can know:

$$
\begin{array}{r}
x(n)=s(n)+w(n) \\
y(n)=\sum_{k=0}^{N} h(k) \cdot x(n-k) \\
e(n)=s(n)-y(n)
\end{array}
$$

So the filtering algorithm's mean square error is:

$$
E\left[e^{2}(n)\right]=E\left\{\left[s(n)-\sum_{k=0}^{N} h(k) \cdot x(n-k)\right]^{2}\right\}
$$

By the principle of minimum mean square error, the value of equation above is the smallest, so Wiener filter's weighting coefficient $h(k)$ should meet the linear equation as follows:

$$
\sum_{k=0}^{N} \varphi_{x x}(m-k) \cdot h(k)=\varphi_{s x}(m) \quad 0 \leq m \leq N
$$

In the equation, $\varphi_{x x}(m)$ is the auto-correlation sequence of the observed signal, $N$ is the wiener filter's order, $\varphi_{s x}(m)$ is the cross-correlation sequence between desired signal and observed signal ${ }^{[9-10]}$.

For Wiener filter, the signal should be stationary. Technically, the vibration signal which is sampled in the industrial scene is non-stationary signal, which cannot directly used by Wiener filtering. However, the vibration signal has a certain stable periodic characteristics. When the signal has a more obvious periodicity, we can use the cyclic Wiener filter to process the data by segmentation and circle. A whole segment of the original signal is divided into a small range by a certain rule, then Wiener filtering is used in each small range in order to form a circle, in later the segmentation data is linear integration. The cyclic Wiener filter is the improvement of Wiener filtering, which has a better filtering effect at the lower order. The simulation result of cyclic Wiener filter is shown as Figure 5:

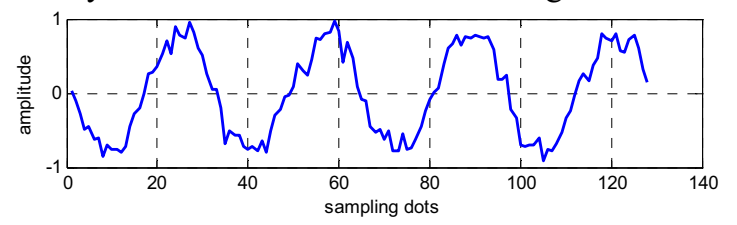

Figure5. Simulation result of cyclic Wiener filter

\section{Cyclic Wiener filtering algorithm in Discrete Cosine Transform}

\author{
5.1 The combination of DCT and cyclic wiener \\ filtering
}

When using DCT filtering, the noise which is added in coefficient preserving data cannot be removed that can be filtered again by Wiener filter to improve the filtering effect. When the length of signal is longer, the energy concentration of DCT transform is reduced, the requirement of Wiener filter's order is also improved, so after segmentation, the Wiener filtering in DCT domain should be considered. Generally speaking, the vibration signal has the characteristic of stationary in cycle, so the vibration signal can be segmented by the whole period, and then each period is transformed into DCT domain then processing by Wiener filtering. It's called cyclic Wiener filtering in DCT domain. This combination not only makes up for the lack of DCT filtering, but also plays the better role of Wiener filtering. The algorithm process of cyclic Wiener filtering in DCT domain is as follow:

(1) The observation signal and ideal signal are segmented respectively according to the same role;

(2) Each segment is for Discrete Cosine Transform respectively

(3) The first segment data by DCT transform is for Wiener filtering, the result is saved and retained.

(4) The upper filtering result is as the next desired signal segment for filtering processing.

(5) A number of segments of the signal which is processed for a simple combination.

6) Inverse Discrete Cosine Transform for recombination data then gets the filtering effect.

\subsection{Filtering result analysis}

Analysis of algorithm principle, DCT filtering is a kind of filtering that has zero phase shift or none phase shift. Choosing the best reserved length can obtain a certainly of filtering effect. Also the characteristic of the waveform signal is not obviously lost. Meanwhile, cyclic Wiener filter is a kind of linear filtering, so this algorithm can achieve a better phase characteristics on the whole.DCT filtering removes the noise of coefficient of superposition, then the cyclic Wiener filtering can further enhance the filtering effect. The segmentation processing, that can greatly reduce the order of Wiener-Holf equation. So the problem mentioned above can be easily resolved.

Simulation result of cyclic Wiener filtering in DCT domain is shown as Figure 6.From the actual simulation results we can see: this algorithm not only achieve the better noise filtering effect, separates the noise and signal in maximum, also the features of the original signal are well preserved, meanwhile the good phase characteristics are obtained.

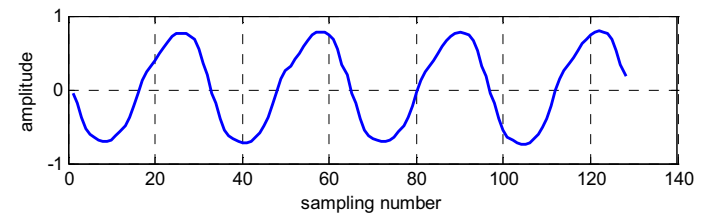

Figure6. Simulation result of cyclic wiener filtering in DCT domain

Practice shows the algorithm that cyclic Wiener filtering in Discrete Cosine Transform Domain can meet the filtering demands for this kind of vibration signal; also it achieves the effective compression for the data which reduces the 
amount of data.

\section{Conclusion}

This paper presents an algorithm for cyclic Wiener filtering algorithm in Discrete Cosine Transform Domain for vibration signal. This algorithm combines the DCT filtering and cyclic Wiener filtering effectively, which achieves the better filtering effect. Simulation result shows that this algorithm can achieve ideal effect for steam turbine's vibration signal denoise processing, and it can ensure the good linear phase, and the filtering processing for vibration signal does not produce obvious phase distortion. Also at the same time to ensure the linear phase characteristics this algorithm achieve the better filtering effect, which performance is better than Wiener filtering algorithm alone or DCT filtering algorithm alone. The algorithm which processes the data in segment, so the order of Wiener filtering is reduced, solves the problems that the energy concentration of long data in DCT domain is low. Using the characteristics of DCT energy concentration, it further reduces the order of computation, meanwhile it realizes the vibration signal data compression, The basic principle of the algorithm can be also applied to other signal denoise processing which have the similar filtering requirements. So it has a very good value and application prospect.

\section{References}

1. ZHANG Jun, WANG Jin-ping, ZHU Bo. Signal Filtering and Feature Extraction Technology of Rotating Machinery Vibration Signal Process. Journal of Nanjing Institute of Technology :Natural Science Edition,3,15,(2009)

2. WANG Jing-fang. On DCT Domain Wiener Filtering for Speech Enhancement. Journal of Hunan International Economics University,1,83,(2014)

3. Ming Yang, CHEN Jin, DONG Guang-ming. Rolling Bearing Fault Diagnosis Based on Cyclic Wiener Filter and Envelop Spectrum. Journal of Vibration Engineering,5,537,(2010)

4. GUAN Bo, Hu Jin-song. Study of the DCT-Based Vibration Signal Zero Phase Distortion Filtering Method in Rotating Machinery. Turbine Technology,4,285,(2007)

5. G.K Singh, Saleh Al Kazzaz Sa'ad Ahmed. Vibration signal analysis using wavelet transform for isolation and identification of electrical faults in induction machine. Electric Power Systems Research, 68,119-136,(2003)

6. WANG Jin-ping, DENG Ai-dong, DING Ke-ke. Research on and Design of DSP-Based Turbine Vibration Signal Acquisition System[J].Power System Engineering,24,49,(2008)

7. CHEN Jia-yuan, ZHANG Qiu-ju, YU Wen-kai. Research on Method for Vibration Signal Compression for a Compressor Based on DCT .Compressor Technology,4,5,(2009)

8. GUAN Bo, HU Jin-song. Research on Reduction Method for Vibration Signals of Rotating Machinery Based on DCT .Wind Turbine
Technology,3,80(2007)

9. Wu Qing-ju, Tian Xiao-bo. Receiver Function Estimated by Wiener Filtering. Earthquake Research in China.17,387-388(2003)

10. W. Gardner. Cyclic Wiener Filtering: Theory and Method. IEEE Transaction on Communications.41,151-163(1993) 